\title{
Language Proficiency Capabilities among International Students in an International University Setting
}

\author{
Sandra Chukwudumebi Obiora, Dr.Nothando Moyo \\ Cyprus International University, Faculty of Economics and Administrative Sciences, Nicosia, Cyprus \\ Email (corresponding author): nmoyo@ ciu.edu.tr
}

\begin{abstract}
In this current day and age, language proficiency and enthusiasm to learn new languages have risen because of several reasons entailing like study, work and business. This phenomenon is even more obvious in international universities where more than half the population of students schooling in that country is foreigners. In many cases, like in the case used for this study; North Cyprus universities, the language used to teach and learn is English language. This therefore entails that on average, almost all students already speak at least 2 languages wherein they speak their own dialects, or cultural languages, while using English language proficiently to communicate with others, learn, study, and write examinations. Thus, in researching this, the aim is to see the extent to which the students are bilingual, or multilingual. Our findings confirm that individual must live, or should have lived in a country in order to proficiently speak that country's language and second, third, or fourth languages are learned during teenage years rather than in one's childhood.
\end{abstract}

Keywords: Language proficiency, International schooling, International communication, International company

\section{Introduction}

Language is one of the key means via which humans communicate. It comes either as spoken, written, or in nonverbal expression. Seeing as human beings have evolved with a pressing need to communicate even more than in the past, language has become more important. Due to the fact that world is becoming a global village, the demand to know more languages as an individual and in different societies continue to rise. As it is stated by Govindarajan (2001), every international company's competitive ability is highly dependent on its ability to coordinate critical resources and information that are spread across different geographical locations overcoming barriers of geography, language and culture. With other words a language-competency greatly impacts communication and communication dynamics (Babcock, 2001) in all aspects of life as well as business. Thus, these days, it is very common for a person to speak more than two languages fluently either by reason of birth and nationality, or due to the need to study and acquire different opportunities to improve in different areas of life.

Thus, enabling Language proficiency refers to a person's ability to use a language for different purposes. This includes the ability to listen, read, write, and speak. Proficiency is commonly measured using guidelines developed by the American council on teaching of foreign languages (ACTFL). It measures proficiency on a scale of novice, intermediate and superior; low, mid, and high. The aim of this research, therefore, is to identify the means, by which people predominantly acquire new languages; whether they lived in that country for some time, or they were born there and as such acquired proficiency in it. Also, we would like to identify how many languages on average an international university student speaks. 
In the business world, the intermixing of different cultures is a complete inevitability. In essence, one's ability to speak, or not to speak a language has a big impact on the outcome of certain activities, deals, processes and interactions. University students, especially, those studying in Business Administration are students who have a wide variety of skills and abilities. More especially, in an international university where the main medium of communication and teaching is English language, the probability of finding international students whose main language is not English rises. It is, therefore, interesting to acquire knowledge and first hand details on the fact when and how language proficiency is acquired.

\section{Methodology}

Hypothesis

The research hypothesizes that an individual must live, or should have lived in a country in order to proficiently speak that country's language.

Defining proficiency

Considering the word proficiency is recurrent in this research, it is best to clarify which form of proficiency the researcher is focusing on as regards language.

- Exercising great competence and facility.

- Skilfulness in the command of fundamentals through familiarity and constant practice.

Defining the problem

Many people have trouble learning and retaining a new language for the following reasons:

- It has a different grammatical conceptual framework to their first language;

- The characters for writing is completely separate from first language;

- The nuances in basic conversation are not in alignment with what is normally used.

Variables under consideration

Before making or drawing out the research questions, it is important to consider the different variables that affect language proficiency. These variables then move to form and narrow down the questions that are generally necessary for approving or disproving the over heading hypothesis of this research. Several of these variables having an impact include:

- Home/ family life;

- Friendships;

- Schooling experience (language of Instruction);

- Age;

- Nationality;

- Language used for thought;

- International exposure;

\section{Acquiring input data}

Secondary and Primary data collection process

Secondary data was generally collected via the internet from prior journals and psychological research done on language proficiency. The primary data needed for the research was collected by narrowing down these different variables into yes or no, and or Likert scales that limit the responder's responses into quantifiable information.

\section{Secondary data information collected}

From prior research already done, it was observed that within the first six years of one's growth, first language is solidly generated. Children learn 3 new words per day within this time (Kluger, 2014). The learning of metaphors, syntaxes, allusions, and grammatical details are understood in this period. First, second and third languages can be attained at about the same time depending on where a person was born and located. A good example of this concept are Nigerian children living in Turkey or China who tend to be trilingual without noticing; speaking and assimilating English, their Nigerian language, and Turkish or Chinese at the same time. 


\section{Analysis}

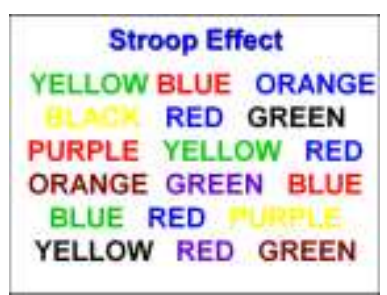

Figure 1: The Stroop effect concept

The Stroop effect is understood to be a phenomenon where the brain reacts slowly when forced to deal with information that conflicts itself. This occurs due to interference resulting from competing functions of our brains (WiseGEEK, 2014 - Ridley 1935). The Stroop effect explained by psychologists and several neurologists as being the same or a similar process to that which the brain engages in order to switch from language to language. It explains bilingualism and trilingualism and such. In essence, one's struggle to speak the right language or say the right word at the right time in the right context when different languages and scenarios seem to clash in our brains.

Primary data collection methodology and responses

The primary data required for this research was done via a questionnaire consisting of 5 questions where respondents were asked:

1. How many languages do you speak?

$$
1-2-3-4-5-6
$$

2. Are you more proficient in your first, second, or third language? First-Second- Third-Fourth

3. At what age group did you become most proficient in your first language? Childhood- Teenage years-Young adulthood-Adulthood

4. At what age group did you become proficient in your second, third or fourth language? Childhood- Teenage years-Young adulthood-Adulthood

5. For your second, third, or fourth language, did you live in that country in order to learn it? Yes- No

Analyzing and testing results

The data collected was then placed onto Excel as a quantitative software system in order to draw accurate conclusions. 
Sandra ChukwudumebiObiora, Dr. NothandoMoyo

Language Proficiency Capabilities among International Students in an International University Setting

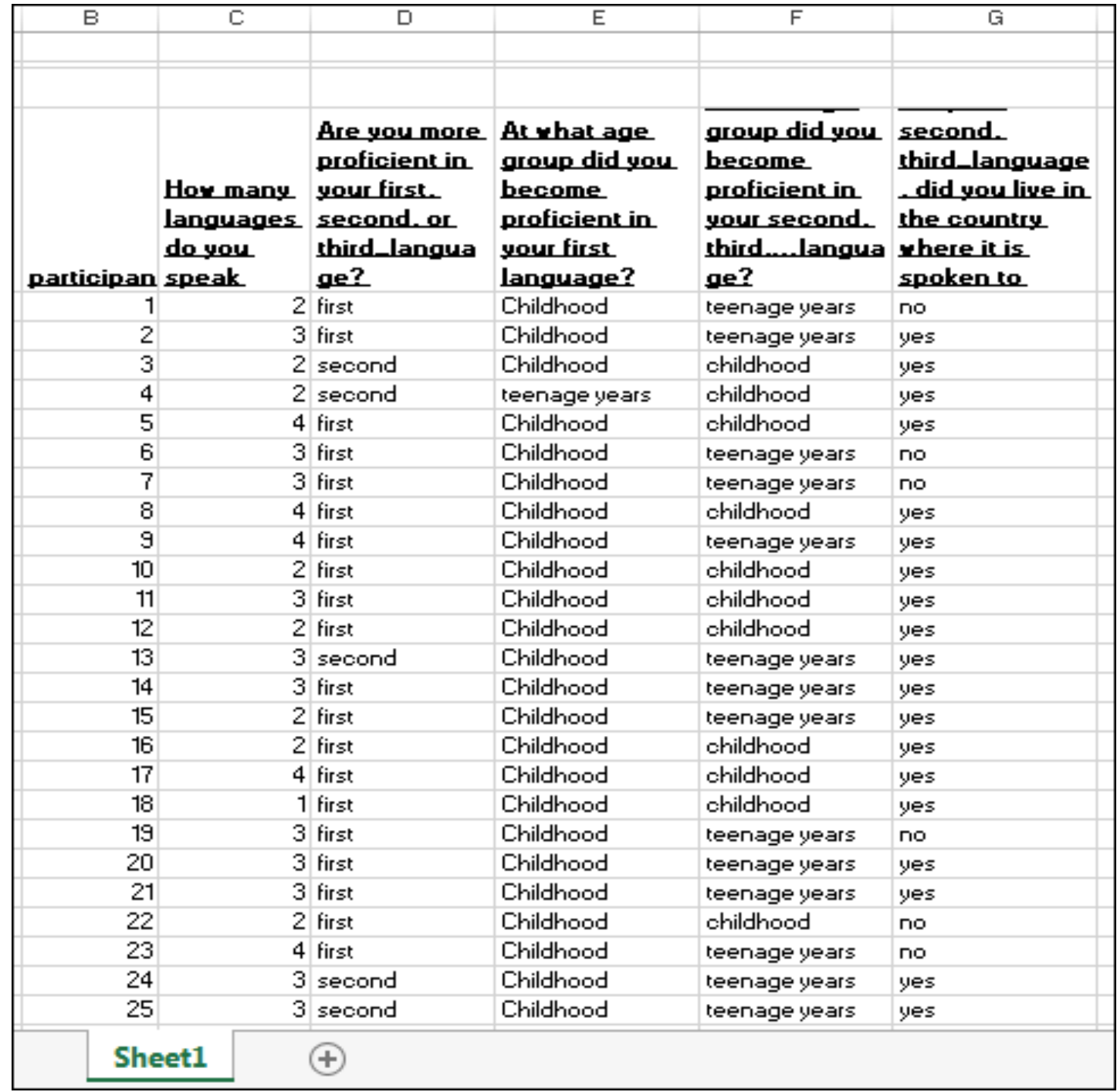

Figure 2: Raw data

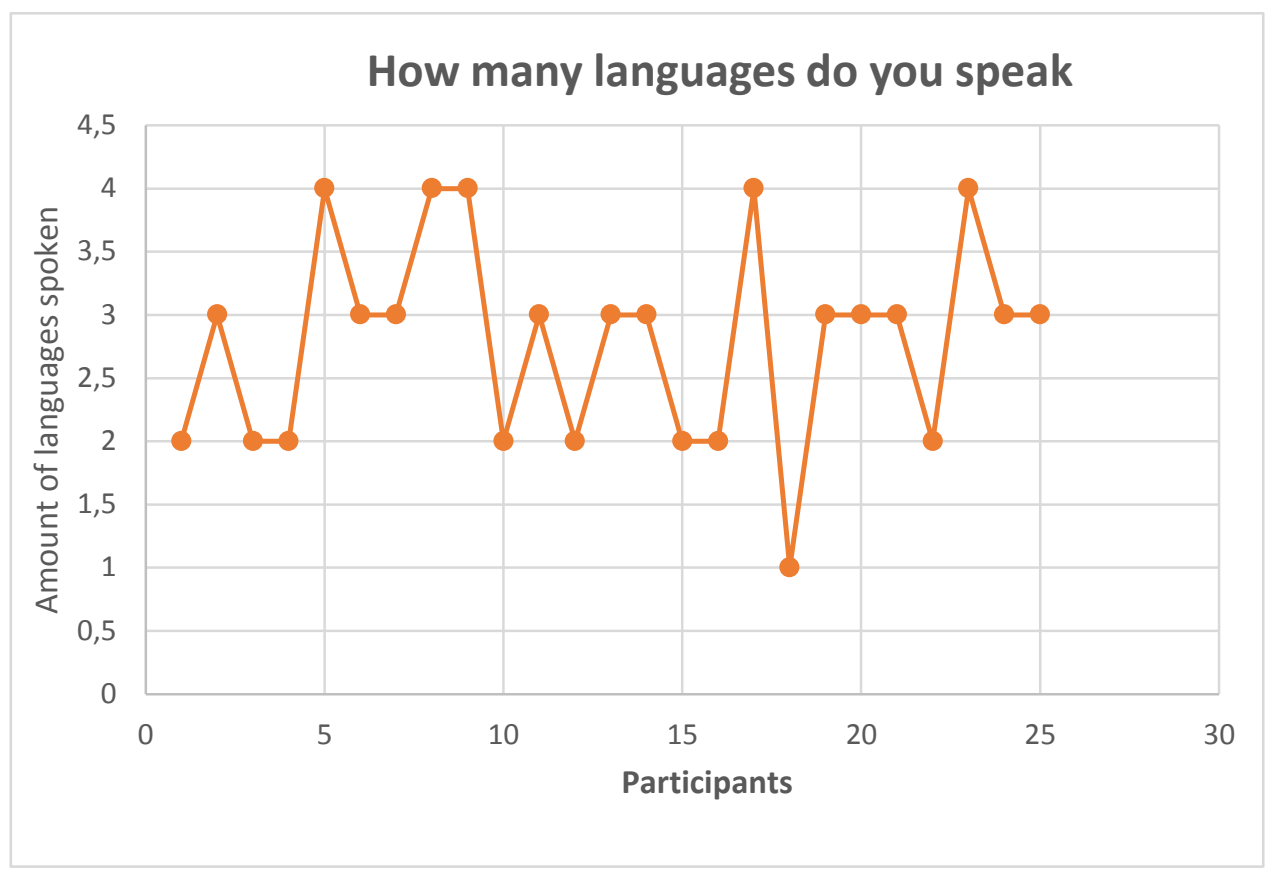

Figure 3: Data finding for question 1 
From the graph above, we see that most of the 25 participants asked speak at least 2 separate languages, and at most 4 , and on average 3 .

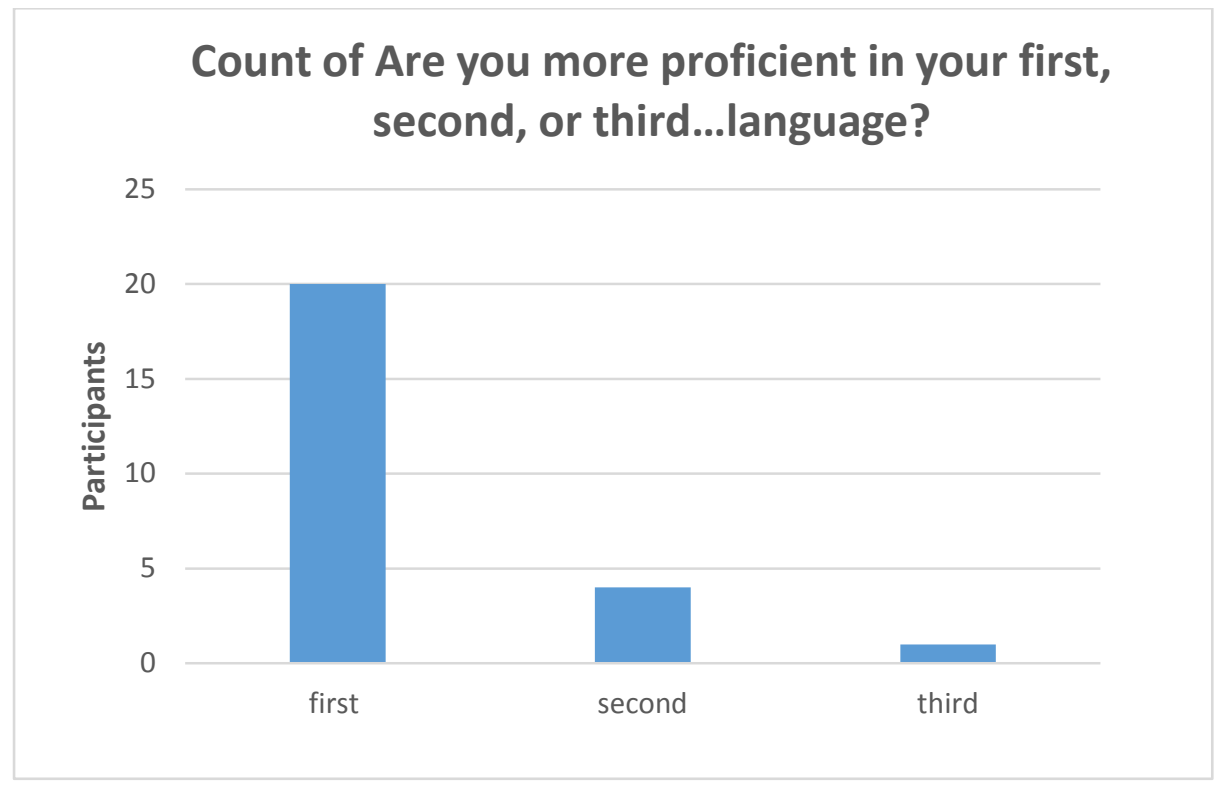

Figure 4: Graph/data finding for question 2

Figure 4 shows us that most of the participants are most proficient in their first language as compared to their second or third.

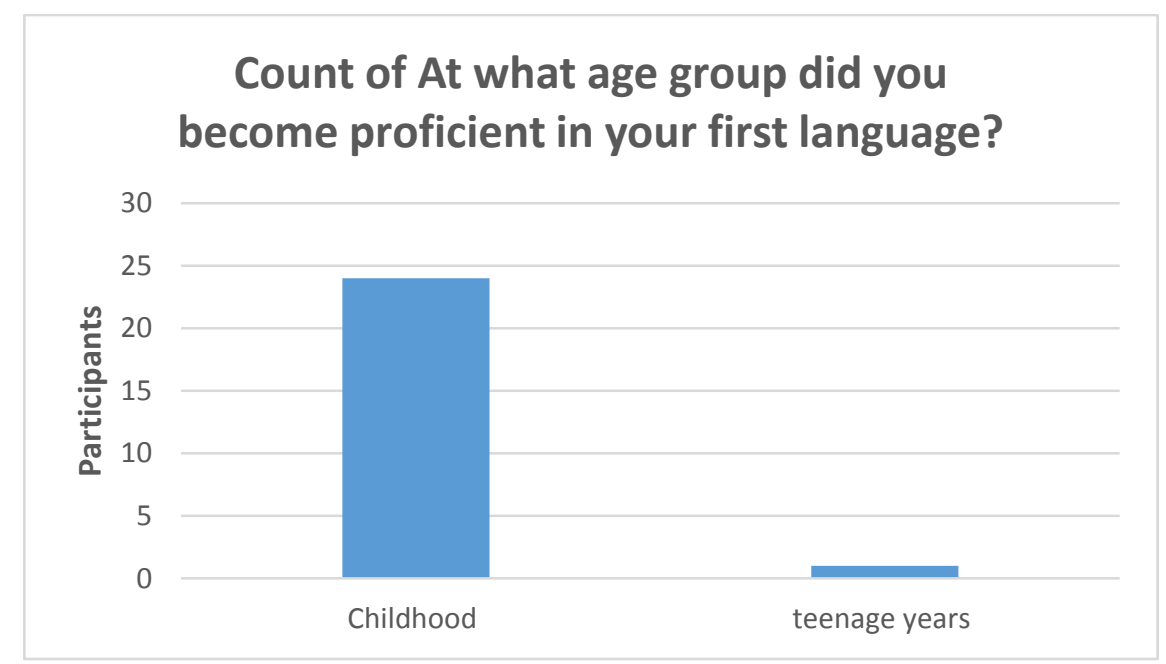

Figure 5: Graph/data finding for question 3

The figure shows that most participants became proficient in their first language during their childhood. 


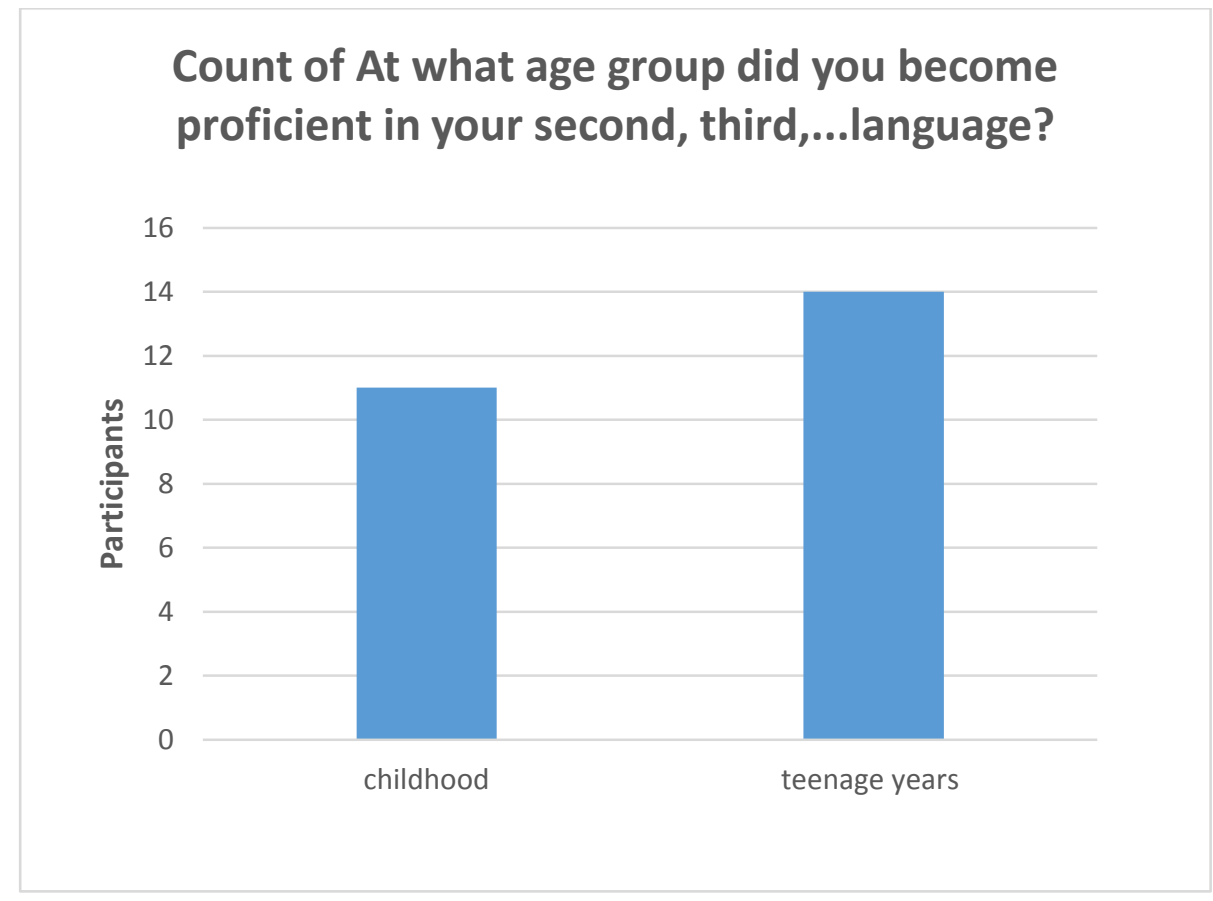

Figure 6: Data findings for question 4

The graph shows that most participants learned or became proficient in their second, third, or fourth language during their teenage years as opposed to in their childhood.

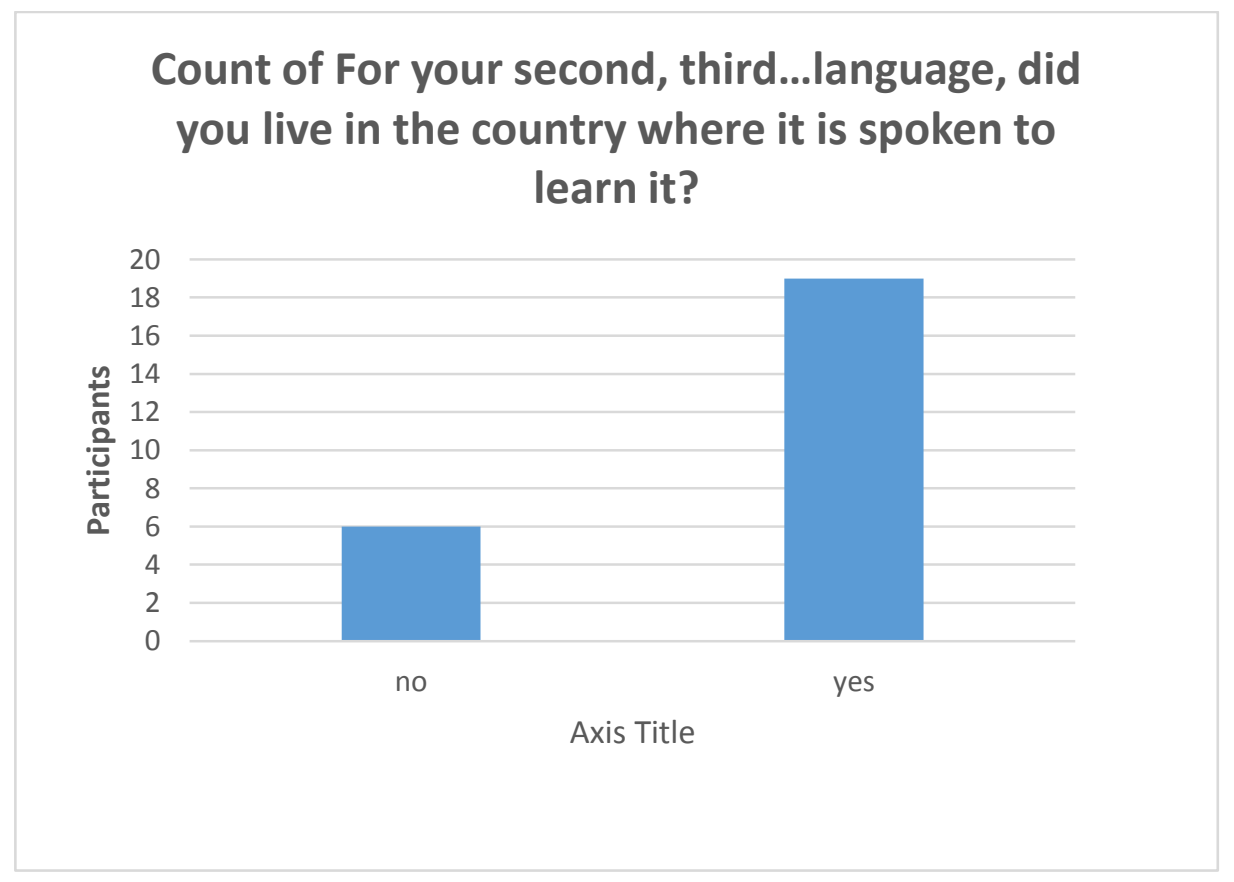

Figure 7: Data finding for question 5

The figure shows that most participants acquired their learned, or second and third languages by living in the country within which these languages are primarily spoken.

Hypothesis confirmed

The hypothesis that an individual must live, or should have lived in a country in order to proficiently speak that countries language, is partially confirmed considering that from the data findings, especially for that of question 4 and 5 , we find that indeed proficiency in second, third, or fourth languages were attained not during childhood, but rather 
during teenage years, and in this time, most of these participants were indeed living in this country in order to become proficient in that country's language as a second, third or fourth language.

\section{Shortcoming of the research}

The research hypotheses are partially approved because there were several limitations to this research which act as very fundamental variables to the approval of the hypothesis. These include:

- Time was insufficient to properly collect data ;

- Sample size was limited and not large enough.

Sample size was focused only on an international school setting.

\section{Findings}

The general findings based on participant's responses for this research include the fact that most people speak an average of 3 languages. They are most proficient in their first language (spoken at home). They acquired proficiency of their first language during their childhood. However, they acquired proficiency in their second, third or fourth language during their teenage years. In which time they lived in that country within which the language is primarily spoken approve the hypothesis that one must have lived in a country to be proficient enough in that language as first, second, third and so on languages.

\section{Conclusion}

We understand that the demand to know more than one language has risen over the years. Most people around the world, especially in international institutions, tend to speak more than one language. This also has implications in the business world as English language (which is not the first language of a vast majority of the countries of the world) is used as the main medium of communication for international business exchanges. We find from this research that the standard in most of these settings is: two languages per person. This is more obvious in International schools, with our focus lying on international universities. In these settings, more than $50 \%$ of the school's population are foreigners to the location and by default they speak two languages; English and their home country's language. In other cases, several of them speak three languages, and in some exceptional cases, speak four or more.

The hypothesis for this research was that a person must have lived in a country for some time, or must have been born there in order to proficiently speak that language. We then defined proficiency as the quality of having great competence, as well as skilfulness of command. Questionnaires were then passed around, and we found that on average, each respondent spoke 3 languages, with higher proficiency levels in their first languages, with many acquiring these languages from childhood, and becoming proficient in their second and third languages during their teenage years and adulthood.

Finally, despite the shortcomings of the research, one key identifiable trait is that the first language was acquired and made proficient through from childhood, while the second, third and fourth languages were necessities that arose and were therefore mastered during teenage years and adulthood.

\section{References}

- Babcock, R.D. and Du-Babcock, B., 2001. Language-based communication zones in international business communication. Journal of Business Communication, 38(4), pp.372-412, CrossRef

- Cartaginese, C. (1992). The Polyglot Project: YouTube Polyglots, Hyper-polyglots, Linguists, Language Learners and Language Lovers, in Their Own Words, United States

- Droop, Mienke, and LudoVerhoeven. "Language Proficiency and Reading Ability in first - and second language Learners." Reading Research Quarterly 38.1 (2003): 78-103. Web, CrossRef

- Fluent U (2014), The 5 Big Advantages to Learning Multiple Languages at Once http://www.fluentu.com/blog/learning-multiple-languages-at-once

- Govindarajan, V. and Gupta, A.K., 2001. Building an effective global business team. MIT Sloan Management Review, 42(4), p.63.

- Kluger. J (2014), Understanding how the brain speaks two languages.www.times.com. 
- Leaver, B. L, and Boris S. (2002) Developing Professional-level Language Proficiency. Cambridge: Cambridge UP, CrossRef

- Li, David C.s. "Towards 'biliteracy and Trilingualism' in Hong Kong (SAR): Problems, Dilemmas and Stakeholders' Views." AILA Review AILA 22 (2009): 72-84.

- $\quad$ Locke, J. L. (1993), The Child's Path to Spoken Language. Cambridge, MA: Harvard UP.

- Macswan, Jeff, and Lisa Pray. "Learning English Bilingually: Age of Onset of Exposure and Rate of Acquisition among English Language Learners in a Bilingual Education Program." BilingualResearchJournal 29.3 (2005): 653-78.

- Mahoney, Kate S., and Jeff Macswan. "Reexamining Identification and Reclassification of English Language Learners: A Critical Discussion of Select State Practices." Bilingual Research Journal29.1 (2005): 31-42. Web, CrossRef

- Nallenart French Curriculum Club (2014). "How Your Brain Learns a New Language."http://nallenart.com/french/how-your-brain-learns-a-new-language

- Web Crawford, J. "Language Choices in the Foreign Language Classroom: Target Language or the Learners' First Language?" RELC Journal 35.1 (2004): 5-20. Web 